\title{
LA MEDITACIÓN DE LA TÉCNICA DI ORTEGA Y GASSET E «'IMPOSSIBILE RITORNO» DI E. ZOLLA
}

\section{THE MEDITACIÓN DE LA TÉCNICA BY ORTEGA Y GASSET AND THE «IMPOSSIBLE RETURN» BY ELÉMIRE ZOLLA}

\author{
PIETRO PIRO* \\ Università di Enna Kore
}

RiASSUNTO: dall'analisi dello scritto del 1933 di Ortega y Gasset, Meditación de la técnica, si compara l'analisi della tecnica di Ortega y Gasset con quella del filosofo e orientalista italiano E. Zolla. Entrambi partono dall'analisi della tecnica come fattore essenziale per comprendere il mondo contemporaneo e il processo di massificazione delle coscienze che essa produce. Tuttavia, i due pensatori propongono soluzioni distinte, più politica e filosofica quella di Ortega, più mistica e «orientale» quella di Zolla.

\section{PAROle CHIAVE: tecnica, l'uomo-massa, mistico.}

ABSTRACT:Analyzing Ortega y Gasset's 1933 text Meditación de la técnica, we compare the analysis of the Ortega y Gasset's technique with that of the Italian philosopher and orientalist E. Zolla. Both depart from the analysis of technology as the key to understanding the contemporary world and the process of the massification of conscience that it produces. However, the two thinkers propose diverse solutions, with Ortega's being more political and philosophical and Zolla's being more mystical and «oriental.»

KEYWORDS: technique, mass-man, mystic.

Questo saggio è dedicato a $G$. Marchianò

*E-mail: sekiso@libero.it 


\section{L'opera di E. Zolla come punto d'irradiazione}

La lettura, lo studio, la meditazione dell'opera di E. Zolla conducono necessariamente ad allargare i propri orizzonti. Il gioco continuo di rimandi, riflessi, irradiazioni, porta a costruire, attraverso un susseguirsi di cerchi concentrici, un mandala personale in cui la coscienza si rischiara a una luce più forte e autentica. Negli studi preliminari alla redazione della voce per il glossario zolliano uomo-massa, (che mi è stata affidata come membro dell'A.I.R.E.Z.), ${ }^{1}$ mi sono imbattuto nella lettura dell'opera del filosofo spagnolo José Ortega Y Gasset, La ribellione delle masse. ${ }^{2}$ In quest'opera per la prima volta compare la definizione di uomo-massa. Dopo averne terminata la lettura (e meditato il contenuto), necessaria per tracciare un quadro cronologico dei lavori che analizzano la massa e le sue relazioni con l'individuo, che precedono la stesura del lavoro di E. Zolla Eclissi dell'Intellettuale, (1959) mi sono imbattuto in un breve lavoro di Ortega, Meditaciòn de la técnica, (1933). Il testo è centrato sulla questione della tecnica e sul suo potere di determinare il destino dell'essere umano e s'inserisce in un ampio dibattito europeo sulla relazione tra tecnica e nichilismo. ${ }^{3}$

\footnotetext{
${ }^{1}$ Sul progetto Glossario Zolliano e sull'A.I.R.E.Z. (Associazione Internazionale di Ricerca Elemire Zolla) vedi il sito www.elemirezolla.org.

${ }^{2}$ J. Ortega Y Gasset, La ribellione delle masse, SE, Milano 2001.

3 "Si pensi alle considerazioni di Werner Sombart su Tecnica e cultura (Technik und Kultur, 1911) o ai Pensieri sulla tecnica (Gedanken über Techik) che Romano Guardini concepì in forma di Lettere dal Lago di Como (Briefe vom Comer See, 1927). Oppure al Lavoratore (Der Arbaiter, 1932) di Ernst Jünger e alla Perfezione della tecnica (Die Perfektion der Tecnik, 1949) del fratello Friedrich Georg, alla Meditazione sulla tecnica (Meditación de la técnica, 1939) di Ortega y Gasset e a L'opera d'arte nell'era della sua riproducibilità tecnica (Das Kunstwerk im Zeitaler ihrer technichen Reproduzierbarkeit, 1936) di Walter Benjiamin. O, ancora, dopo al guerra, alla critica della razionalità tecnologica di Adorno, Horkheimer e Marcuse, e a quella opposta, ma complementare di Heiddegger. E poi L'anima nell'età della tecnica (Die Seele im tecnischen Zeitalter, 1949 e1957) di Arnold Gehel, La tecnica il rischio del secolo (La tecnique ou l'enjeu du siècle, 1954) di Jacques Ellul, L'uomo è antiquato (Die Antiquiertheit des Menschen, 1956-80) di Günther Anders o ancora Il principio responsabilità (Das Prinzip Verantwortung, 1979) di Hans Jonas. Fino a Techne. Le radici della violenza (1979) di Emanuele Severino. Con la loro incisività e radicalità, tutte queste analisi hanno segnato l'emergere del problema». Cfr. F. Volpi, Il Nichilismo, Laterza, Roma-Bari 2003, p. 148.
} 


\section{La lezione come dramma}

Il testo Meditaciòn de la técnica $a^{4}$, raccoglie una serie di lezioni tenute da Ortega nell'estate del 1933 presso l'Università estiva di Santander. Subito Ortega precisa che cosa sia per lui una lezione:

una lezione è questo: incontrarsi da vicino uno con l'altro e legarsi con voi, scontrarsi con voi, scontrarsi con l'effetto positivo o negativo, però sempre serio. Una lezione è una peripezia di forte drammaticità sia per chi la da sia per chi la riceve. Quando questo non avviene, non è una lezione è un'altra cosa-a volte un crimine -perché è un'ora persa e la vita è un tempo limitato e perderne un pezzo è uccidere la vita. ${ }^{5}$

Una lezione è un momento drammatico, in cui si mettono in discussione le verità soggettive e insieme si perviene a una nuova forma di coscienza. Questo non avviene però nelle Università, che subiscono una decadenza inesorabile e che sono diventate dei luoghi astratti e spettrali, proprio a causa del sapere tecnico. La divisione tra Università tecniche e Università umanistiche ha portato l'Università a una decadenza fulminea. Nel Medioevo, l'Università non doveva occuparsi in maniera specifica della tecnica perché il suo spessore non era così elevato da occupare una pedagogia a essa riservata e perché la vita extrauniversitaria poneva sufficientemente in contatto l'uomo con la tecnica semplice e trasparente del tempo. Oggi le cose sono cambiate e il sapere tecnico ha assunto un tale spessore che necessita una vera e propria formazione pedagogica specifica per essere conosciuto. Nonostante si sia giunti nel tempo alla creazione di accademie tecniche.

La collocazione dell'uomo attuale di fronte alla propria vita è più irreale e incosciente dell'uomo medievale e possiede minori cognizioni rispetto alle condizioni nelle quali si svolge la propria vita. ${ }^{6}$

\footnotetext{
${ }^{4}$ Tutte le citazioni sono tratte da: J. Ortega Y Gasset, Meditaciòn de la técnica y otros ensayos, Revista de Occidente, Madrid 1977.

${ }^{5}$ Cfr. J. Ortega Y Gasset, Meditaciòn de la técnica y otros ensayos, Revista de Occidente, Madrid 1977, p. 15 .

${ }^{6}$ Ivi. p. 19.
} 
L'Università dunque, proprio per le contraddizioni che la tecnica le pone dinanzi, necessita per Ortega di una profonda riforma, in grado di affrontare le sfide che la tecnica continuamente pone all'uomo di oggi.

\section{La tecnica definizione e caratteristiche}

Ortega definisce tecnica: «I cambiamenti che l'uomo impone alla natura in vista della soddisfazione dei propri desideri». ${ }^{7} \mathrm{E}$ atti tecnici:

Non sono quelli con i quali l'uomo soddisfa le necessità che le circostanze o la natura gli pongono, sono piuttosto quelli che portano a riformare queste circostanze eliminando per quanto è possibile le necessità, sopprimendo o diminuendo il caso e lo sforzo che necessita per soddisfarle. ${ }^{8}$

La tecnica consiste nell'adattare i mezzi al soggetto. Per l'uomo la necessità fondamentale consiste nel vivere, nel perdurare. Ė vero, però, che vivere non significa la mera soddisfazione dei bisogni elementari, l'uomo infatti vuole vivere bene, soddisfare dei bisogni di benessere e di ben vivere. Le necessità date dalla propria appartenenza biologica (fame, sete, freddo, caldo, sonno) non sono sentite come proprie e ciò che veramente conta è soddisfare bisogni di benessere, bisogni superflui:

Non ci sono dubbi: l'uomo è un animale per il quale solamente ciò che superfluo è necessario. ${ }^{9}$

La tecnica è la produzione del superfluo. Uomo, tecnica e benessere sono essenzialmente sinonimi. La produzione tecnica essendo inscritta inscindibilmente nella sfera del desiderio di benessere, cambia come cambiano i desideri umani. L'idea di un progresso è stata utilizzata in maniera errata perché presuppone un uomo dotato sempre dello stesso tipo di desiderio e che vuole sempre le stesse cose. Il desiderio dell'uomo cambia continuamente e la tecnica non può

\footnotetext{
${ }^{7}$ Ivi. p.32.

${ }^{8}$ Ivi. p. 35 .

${ }^{9}$ Cfr. J. Ortega Y Gasset, op.cit. pag. 39.
} 
che evolversi in maniera proteiforme, cambiando continuamente. Si possono riassumere in atti tecnici ${ }^{10}$ quelli che:

a) Assicurano la soddisfazione delle necessità elementari.

b) Permettono di soddisfare questi bisogni con il minimo sforzo.

c) Creano possibilità completamente nuove producendo oggetti che non esistono in natura. Che permettono di navigare, volare, parlare con luoghi lontani attraverso il telegrafo e le comunicazioni radio.

d) Producono sicurezza. La preoccupazione, l'angustia, il terrore che l'insicurezza provoca, sono uno sforzo che la natura impone all'uomo.

\section{La condizione umana: una lotta per l'esistenza}

La tecnica rappresenta dunque il tentativo dell'uomo di alleviare le sofferenze della propria condizione. L'uomo non si sente totalmente immerso nella natura:

Quello che possiede di naturale si realizza da sé, non è in questione. Ma allo stesso tempo, non lo sente come il suo autentico essere. ${ }^{11}$

Ortega definisce la condizione dell'essere umano: tremenda e senza pari, l'uomo è un ente imperfetto e desiderante:

Un ente il cui essere consiste, non in quello che già è, piuttosto in quello che ancora non è, un essere che consiste in ciò che ancora non è. La stella è quella che già è, né più né meno. Tutto quello che esiste, consiste nell'essere quello che già è, e con il quale, pertanto, coincide naturalmente, —la sua potenzialità con la sua realtà- quello che può essere con quello che è, in effetti, chiamata cosa. La cosa ha il suo essere determinato e realizzato. L'uomo non è una cosa, piuttosto una pretesa, pretesa di essere questo o quello. ${ }^{12}$

\footnotetext{
${ }^{10}$ Ivi., p. 48.

${ }^{11}$ Ivi., p. 56.

${ }^{12}$ Ivi., p. 57.
} 
Pertanto l'uomo, nella sua apertura alla realtà, nella sua infinita possibilità di realizzare i propri desideri, mostra tutta la drammaticità della propria condizione di essere indeterminato che, a differenza degli altri esseri, si ritrova continuamente in scacco, perché la sua condizione non gli permette riposo e pace. Ognuno di noi cerca di realizzare un "programma» che lo porti a completare se stesso nell'immagine che desidera realizzare, nella figura in cui intende definirsi. La relazione tra programma e mondo si caratterizza come una continua lotta drammatica:

Corpo e anima sono cose, ed Io non sono una cosa, bensì un dramma, una lotta per poter essere quello che desidero essere. La pretesa o programma che vogliamo essere, preme con il suo peculiare profilo il mondo intorno, e questo risponde a questa pressione accettandola o respingendola, e decide, di facilitare la nostra pretesa in un punto o metterla in difficoltà in un altro. ${ }^{13}$

Il mondo, dunque, rappresenta per noi la scena in cui forze contrapposte (positive e negative) si relazionano con la nostra impostazione progettuale. La vita è dunque primariamente uno spazio in cui si progetta, si fabbrica per realizzare le proprie ambizioni e dove la contemplazione, il pensiero, la teoria, nascono in seguito. A differenza dell'animale che può vivere tutta la sua vita realizzando i suoi bisogni immediati, l'uomo può vivere solamente se può trascenderli e realizzare "progetti». La missione fondamentale della tecnica sarà: «Dare spazio all'uomo per poter essere libero di godere di se stesso». ${ }^{14}$ Uomo e mondo rappresentano una dualità irriducibile e la tecnica funziona da mediatrice tra le pretese dell'uno e le condizioni di equilibrio dell'altro.

\section{Due tipi di progetto umano II Bodhisattva e il Gentleman}

Se la tecnica rappresenta un mezzo per realizzare i propri progetti e la condizione dell'uomo è così indeterminata che sempre si ripete la tensione tra esse-

\footnotetext{
${ }^{13}$ Cfr. J. Ortega Y Gasset, op.cit. p. 58.

${ }^{14}$ Ivi., p.64.
} 
re e voler essere, allora comprendiamo l'importanza che ha il progetto che l'uomo intende realizzare per un successivo sviluppo dei mezzi tecnici:

Il popolo in cui predomina l'idea che il vero essere dell'uomo si realizza nell' essere un Bodhisatva non può creare una tecnica uguale a un altro in cui si aspira a essere gentleman. Essere Bodhisatva significa innanzitutto, credere che esistere in questo mondo di mere apparenze è non esistere veramente. La vera esistenza consiste per lui, non nell'essere un individuo, frammento dell'universo, piuttosto è maggiormente desiderabile fondersi con il Tutto e scomparire in Lui. Il Bodhisatva, dunque, aspira a non vivere o a vivere il meno possibile. Ridurrà la sua nutrizione al minimo; pessimo per la tecnica alimentare! Cercherà l'immobilità massima, per rifugiarsi nella meditazione, unico veicolo che permette all'uomo di arrivare all'estasi e di decidere di mettersi durante la vita fuori del mondo. Non è verosimile che inventi un'automobile un uomo che non desidera muoversi. Allo stesso tempo, la sua scelta gli permetterà di sviluppare tante tecniche molto distanti da noi europei, come quelle dei fakiri e degli yogi, tecniche dell'estasi, tecniche che non producono cambiamenti nella natura delle cose materiali, bensì nel corpo e nella psiche dell'uomo. Per esempio le tecniche d'insensibilità e la catalessi e le tecniche di concentrazione. ${ }^{15}$

Ortega utilizza questo esempio (lo considera estremo, contro natura) per rivelare la natura profonda del legame tra idea di Sé e della vita, e la tecnica che si dispiega, di conseguenza, da queste premesse. L'idea di Ortega del Bod-

${ }^{15}$ Ivi., pp. 70-71.

${ }^{16}$ L'idea del Bodhisattva che Ortega utilizza come esempio di totale rinuncia alla vita e alla conseguente estinzione dei desideri, è legata alla dottrina del Buddhismo Antico (Hinayana) e non tiene conto dell'evoluzione storica della «missione» del Bodhisattva nel Mahayana, molto più orientata alla vita e all'azione liberatrice. Per un primo approccio:

H. Dayal, The bodhisattva doctrine in Buddhist Sanskrit literature, Motilal Banarsidass, Dehli 1970; Kamamura L.K., (a cura di), The Bodhisattva doctrine in Buddhism, Canadian Corporation for Studies in Religion by Wilfred Laurier University Press, Ontario 1981; Wallace A., -Wallace B.A., (a cura di) A guide to the Bodhisattva way of life = Bodhicaryavatara / by Santideva; translated from the Sanskrit and Tibetan by Vesna, Snow Lion Publications, Ithaca 1997; Wogihara H., Bodhisattvabhumi: a statement of whole course of the Bodhisattva (being fifteenth section of Yogacarabhumi), Sankibo Buddhist Book Store, Tokio 1971; Dwijendra Nath B., Iconography of the Bodhisattva (Bosatsu) images in Japan and India: a comparative study, Calcutta, Centre of Japanese Studies, Calcutta 1991; Katz N., Buddhist images of human perfection: the arahant of the Sutta pitaka compared with the Bodhisattva and the Mahasiddha, Motilal Banarsidass, Delhi 1982; Wendelgard G., Das Bodhisattva-Ideal im Mahayanabuddhismus und seine Verwirklichung im Leben des 
hisattva ${ }^{16}$ è legata certamente alle figure del buddismo monastico del piccolo veicolo e non prende in considerazione gli sviluppi più orientati alla vita del buddhismo Mahayana. Tuttavia, egli coglie un aspetto essenziale del problema: la natura inscindibile tra progetto del Sé e progettazione tecnica. Dal lato opposto, la figura del gentleman come tipo umano rappresenta l'esatto contrario del Bodhisattva. Egli, infatti, cerca di realizzare nel mondo e attraverso le strade del mondo tutti i propri desideri. Egli accetta la lotta per l'esistenza come elemento sostanziale che caratterizza la vita, accetta la lotta economica, sociale e politica. L'uomo si realizza attraverso la lotta. Certamente ad inventare questo modo peculiare di essere uomini sono stati gli aristocratici inglesi che, però, si sono chiusi troppo e hanno posto troppi vincoli di accesso alle professioni alle quali si sono dedicati, ponendo così un limite molto forte al proprio sviluppo. Il gentleman, non può accettare limitazioni alla realizzazione del proprio Sé e quindi, per sua natura, egli aspira alla libertà assoluta, intesa come possibilità illimitata di realizzare i propri desideri:

Il gentleman in opposizione al Bodhisatva, vuole vivere con intensità in questo mondo e nutrire la propria individualità il più possibile, concentrasi su se stesso e nutrire la sensazione d'indipendenza di fronte a tutto (...) Il gentleman non è un intellettuale. Cerca il decorum in tutta la sua vita: anima pulita e corpo pulito. Però, chiaramente, tutto questo presuppone ricchezza; e l'ideale del gentleman portò, in effetti, a creare un'immensa ricchezza. ${ }^{17}$

ladakhischen Volkes P. Lang,; New York 1991; Leighton T.D., Bodhisattva archetypes: classic Buddhist guides to awakening and their modern expression, Penguin Arkana, New York 1998; Sangharakshita B., The Bodhisattva ideal: wisdom and compassion in Buddhism, Windhorse, Birmingham 1999; Mrozik S., Virtuous bodies: the physical dimensions of morality in Buddhist ethics, Oxford University Press, New York 2007; Gioi Huong B., Bodhisattva and Sunyata in the early and developed Buddhist traditions, Eastern Book Linkers, Delhi 2004; Cantideva, Bodhisattvacaryavatara: guida allo stile di vita di un Bodhisattva, Edizioni Istituto Lama Tzong Khapa, Pisa 1983; Sangharakshita B., The bodhisattva: evolution and self-transcendence, Windhorse Publications, Glasgow 1983; Dessi U., Il bodhisattva Kannon nel buddhismo giapponese: testi e studi, L. Giordano, Cosenza 2007; Ci Y., The Bodhisattva ideal in selected Buddhist scriptures (its theoretical and practical evolution), School of Oriental and African studies, London 2005; Pagel U., The Bodhisattvapitaka: its doctrines, practices and their position in Mahayana literature, Institute of Buddhist Studies, Tring U.K. 1995.

${ }^{17}$ Cfr. op.cit. pp.79-80. 


\section{L'Hidalgo}

Ortega medita un tipo esemplare di vita da contrapporre al gentleman, un tipo di vita che sia compatibile con la miseria che continuamente minaccia il nostro pianeta. Egli lo individua nell'Hidalgo (lett. persona che appartiene a una classe nobile e distinta per discendenza di sangue, s'intende anche la persona nobile per animo generoso e valoroso ${ }^{18}$ ). La differenza più evidente con il gentleman è che:

L'hidalgo non lavora, riduce all'estremo le sue necessità materiali e, di conseguenza, non crea tecnica. Vive alloggiato nella miseria come le piante del deserto che sanno vegetare senza umidità. Però non è meno determinato a dare alla propria condizione una soluzione degna. ${ }^{19}$

Ortega ha dedicato la sua prima opera del 1914, Meditaciones del Quijo$t e^{20}$, all'analisi dell'Hidalgo più famoso della letteratura mondiale: Don Quijote de la Mancha. In quest'opera di Cervantes (fondamentale per tutto il pensiero orteguiano), la questione del progetto personale assume una funzione decisiva. Secondo la celebre espressione di Ortega, che è poi diventata una sorta di manifesto: «Io sono io e la mia circostanza e se non salvo la mia circostanza non salvo me», ciò che più è messo in evidenza, è l'importanza del progetto (in questo caso il progetto di filosofare) come possibilità di salvezza. La vera salvezza non è quella di Don Quijote che spinto dai suoi ideali cavallereschi progetta una lotta contro i mulini a vento, la salvezza e quella di Cervantes che, trovandosi in un'epoca (circostanza) senza via d'uscita, riuscì a creare un'opera in grado di diventare un punto di riferimento per l'intera umanità, per profondità di spirito e acutezza di riflessione filosofica e che allo stesso tempo, mantiene vive tutte le peculiarità dell'uomo mediterraneo. Un'opera che in qualche modo, ha salvato l'autore e le sue circostanze. ${ }^{21}$ Dice

\footnotetext{
${ }^{18}$ Cfr. Diccionario esencial de la lengua española, Real Academia Espanola, Madrid 2006, p.772.

${ }^{19}$ Cfr. José Ortega Y Gasset, Meditaciòn de la técnica y otros ensayos, Revista de Occidente, Madrid 1977 p.81

${ }^{20}$ Cfr. J. Ortega Y Gasset, Meditaciones del Quijote, ed Julian Marias, Catedra, Madrid, 1984. Per l'edizione italiana vedi: J. Ortega y Gasset, Meditazioni del Chisciotte, (tr.it.) Arpaia B., Guida, Napoli 2000.

${ }^{21}$ «Lidea principale dell'etica orteguiana, che si sviluppa nei testi degli anni 20 , è già presente qui: la morale come sforzo e magnanimità fondata nella radicale libertà dell'essere umano; la necessità di
} 
Ortega: "Questo è per me il vero quijotismo: quello di Cervantes, non quello di don Quijote».22

Con questa presa di posizione si distanzierà da coloro che (come M. De Unamuno) intendono interpretare la figura del Don Quijote come l'esempio da seguire per risolvere i problemi dell'uomo moderno e che, per le circostanze poste dalla tecnica, deve trovare una strada nuova, una strada legata all'azione e, nel caso di Ortega, all'azione del filosofare.

\section{Gli stadi della tecnica}

Per individuare perché la tecnica occupi uno spazio così rilevante nella vita dell'uomo di oggi, Ortega cerca di ricostruire una storia a tappe ${ }^{23}$ del rapporto tra uomo e tecnica, distinguendo tre stadi:
A) La tecnica del caso
B) La tecnica dell'artigiano
C) La tecnica del tecnico

creare in accordo con la circostanza nella quale l'uomo si trova — che è sempre concreta—, la regola dell'azione, anticipando di molti anni quello che gli esistenzialisti chiameranno «morale della situazione», sebbene senza coincidere con loro nell'assenza di regole. Al contrario, Ortega si avvicina ad Aristotele, del quale prenderà come marchio etico e vitale, la sua famosa immagine dell'arciere che deve centrare il bersaglio». Cfr. J. Lasaga Medina, José Ortega y Gasset (1883-1955). Vida y filosofia, Biblioteca Nueva, Madrid 2003, p.48.

${ }^{22}$ Cit. in J. Lasaga Medina, op.cit. p. 43. In questa presa di posizione c’è un chiaro riferimento all'opera di M. De Unamuno, (pubblicata un anno prima) Del sentimento trágico de la vida, e una sostanziale differenziazione, per Unamuno infatti: «Tuttavia, l'eroe del pensiero spagnolo non dobbiamo cercarlo forse in un filosofo realmente vissuto in carne e ossa, ma in una entità poetica e di azione, più reale di tutti i filosofi: in Don Chisciotte. Poiché esiste un donchisciottismo filosofico, senza dubbio ma esiste anche una filosofia donchisciottesca. Ed è forse diversa, nella sostanza, dalla filosofia dei conquistadores, dei fautori della Controriforma, di Loyola, e soprattutto — nella categoria del pensiero astratto ma sentito - dei nostri mistici?». Cfr. M. De Unamuno, Del sentimento tragico della vita, (tr.it.), Lopez y Garcia-Plaza J., Piemme, Casale Monferrato 2004, p.296.

${ }^{23}$ Cfr. J. Ortega Y Gasset, op.cit. pp.91-118. 


\section{A) Tecnica del Caso}

La tecnica del caso è quella dell'uomo primitivo e preistorico, egli ignora la tecnica come tecnica; non si rende conto di possedere tra le sue capacità quella che gli permette di riformare la natura secondo i propri desideri. Il repertorio degli atti tecnici dell'uomo primitivo è scarso e non arriva a formare un corpo sufficientemente voluminoso perché si possa differenziare sostanzialmente dal repertorio degli atti naturali. Sconosce completamente il carattere essenziale della tecnica, che consiste nell'essere una possibilità di cambiamento e di progresso, inizialmente, illimitato. Gli atti tecnici sono esercitati da tutti i membri della collettività e nessun atto tecnico si discosta dal suo substrato magico. Ogni invenzione gli appare come una manifestazione della forza della natura.

\section{B) Tecnica dell'artigiano}

È la tecnica dell'antica Grecia, della Roma pre-imperiale e del Medio Evo. Il repertorio di atti tecnici è cresciuto enormemente. Quest'acquisizione favorisce la chiara coscienza della tecnica come funzione generica e illimitata. Nell'artigianato, che si trasmette in scuole e attraverso l'apprendistato, lo strumento tecnico, l'utensile, è solamente un supplemento dell'uomo. L'uomo rimane l'attore principale. La macchina al contrario diventa lo strumento in primo piano e non è la macchina che aiuta l'uomo, semmai il contrario: è l'uomo che aiuta la macchina. L'artigiano è chi realizza il progetto e lo mette in opera. L'artigiano è allo stesso tempo tecnico e operaio. La dissociazione tra tecnico e operaio è uno dei sintomi principali del terzo stadio.

\section{c) Tecnica del tecnico}

È la tecnica del mondo di oggi. Si caratterizza per un illimitato repertorio di atti tecnici i quali hanno superato il repertorio degli atti naturali e senza i quali l'uomo non riesce più a vivere. L'uomo nasce in un paesaggio di oggetti tecnici che offusca la scena naturale al punto tale che gli oggetti tecnici non sono più percepiti come tali (quindi fabbricati) ma vissuti come naturali. L'uomo può arrivare a perdere la coscienza delle condizioni morali con le quali questi mezzi tec- 
nici sono prodotti e regredire a semplice aiuto per le macchine. Si crea la figura pura del tecnico: l'ingegnere. Questi uomini si dedicano esclusivamente alla tecnica senza preoccuparsi se i fini che si propongono di realizzare sono compatibili con i mezzi a disposizione. La tecnica assume un valore assoluto e un potere quasi illimitato.

\section{Il sogno di Ortega: un corso sulle tecniche dell'anima in Oriente}

In questa ricostruzione Ortega dimostra l'attitudine fondamentale dell'essere umano che è quella, a suo avviso, di modificare l'ambiente in funzione delle proprie esigenze progettuali. L'immensa proliferazione degli atti tecnici nasconde l'immenso desiderio, proprio di ognuno, di realizzare il proprio progetto e di gettare nel mondo le proprie condizioni e le proprie regole. Gli oggetti tecnici non sono altro che desideri di realizzazione concretizzati, idee cristallizzate in forma di cosa. Non c'è dunque speranza di un ritorno al passato, perché i desideri dell'uomo sembrano sempre più illimitati e gli sforzi per soddisfarli altrettanto illimitati. In quest'ottica pessimistica, ${ }^{24} \mathrm{che}$ non conosce vie di fuga, Ortega termina il suo breve saggio con un'apertura a Oriente:

La vita umana non è solamente lotta con la materia, quanto piuttosto, lotta dell'uomo con la sua anima. Che cosa possono dimostrare l'America e L'Europa come repertorio di tecniche dell'anima? Non è stato, in quest'ordine di cose, superiore l'Estremo Oriente? Da qualche anno sogno un possibile corso in cui si mostrano l'uno di fronte all'altro le tecniche de l'Occidente e le tecniche dell'Asia. ${ }^{25}$

Ci pare che Ortega abbia saputo cogliere la sfumatura, sottile ma sostanziale, tra tecnica Occidentale e tecnica Orientale. L'Oriente, infatti, pur aven-

\footnotetext{
${ }^{24}$ «Nella Meditazione della tecnica, (1933) Ortega radicalizza la sua visione, sempre massimamente pessimista, della vita umana — ricordare: è dramma e naufragio—, quando afferma che l'uomo è un errore: non che l'uomo sta o vive nell'errore o è condannato a soffrire, piuttosto le si attribuisce la condizione metafisica dell'essere errore». Cfr. J. Lasaga Medina, op.cit. p. 48

${ }^{25}$ Cfr. J. Ortega Y Gasset, op.cit. p.118.
} 
do prodotto (soprattutto nell'antichità) una tecnica straordinariamente avanzata (la Cina ha inventato moltissime cose con largo anticipo sull'Occiden$\left.\mathrm{te}^{26}\right)$ ha saputo sviluppare anche una tecnica dell'anima che riuscisse ad affrontare e a risolvere i problemi interni dell'essere umano, che lo tormentano quanto (e forse più) di quelli esterni. Quando la tecnica occidentale ha fornito a una grande quantità di persone, la possibilità di soddisfare i propri bisogni, un ospite inquietante ha cominciato ad aggirarsi nelle case degli occidentali, un ospite la cui natura disgregatrice ancora non ha pienamente dimostrato tutta la sua potenza: il nichilismo. Infatti, oggi:

Il processo planetario della razionalizzazione scientifico-tecnica ha portato alla soluzione di intere serie di problemi. Eppure, a fronte dei loro successi, la scienza e la tecnica sono incapaci di produrre esperienze simboliche di senso in cui inscrivere il nostro essere nel mondo e nella storia. Anzi le trasformazioni che esse hanno prodotto accelerano il disincanto e la crisi dei fondamenti, cioè l'erosione e la dissoluzione dei quadri di riferimento tradizionali. Si è così aperta una frattura sempre più profonda fra l'homo faber e l'homo sapiens, tra ciò che l'uomo sa e può fare e la sua capacità di valutare ciò che è ragionevole fare. ${ }^{27}$

L'uomo della tecnica è stretto nella morsa tra ciò che può fare e ciò che è giusto fare e questa morsa lo lacera e lo condiziona e, in fin dei conti, lo limita nel suo sviluppo spirituale. È per questo motivo che Ortega, pur ponendo al centro della sua antropologia l'Io, allo stesso tempo, si rende conto che la scelta progettuale del Bodhisattva rappresenta una possibilità diversa e contrapposta a quella del gentleman. A livello personale Ortega propende verso l'ideale dello hidalgo e della filosofia come salvezza della propria circostanza e ritiene che più vicino all'ideale del Bodhisattva sia stato, con la sua opera, E. Zolla.

\footnotetext{
${ }^{26}$ Sulla capacità della Cina antica di anticipare di moltissimi anni la scienza occidentale, si veda per iniziare

J. Needham, Scienza e Civilizzazione in Cina.1. Lineamenti introduttivi, Einaudi, Torino 1981.

${ }^{27}$ Cfr. F. Volpi, Il Nichilismo, Laterza, Roma-Bari 2009, pp.152-53.
} 


\section{Tecnica e massificazione nell'Eclissi dell'intellettuale di E. Zolla}

Nell'opera del 1951 edita da Bompiani, Eclissi dell'Intellettuale, Zolla ha saputo dimostrare la relazione inscindibile che esiste tra utilizzo della tecnica e massificazione. L'opera muove il suo lavoro critico dalla messa in discussione dell'affermazione secondo la quale:

L'industria e il macchinismo possono si danneggiare lo spirito, ma ciò dipende solo dal loro cattivo uso. ${ }^{28}$

Quest'affermazione tradisce, da un lato, la credenza generalmente diffusa da parte degli uomini moderni di poter dominare la tecnica a proprio piacimento, dall'altro la convinzione che la tecnica possieda solamente un valore d'uso e non un valore di per sé e quando uno strumento non è utilizzato esso, per così dire, riposa nell'essere della propria neutralità. Entrambe queste credenze sono false. L'uso della tecnica non è neutrale, l'uso stabilisce rapporti mediati che subiscono il potere d'influenza del mezzo tecnico, alterandoli e modificandoli. Lo strumento tecnico stabilisce nuove gerarchie di valori e introduce categorie sociali nuove, in grado di assumere ruoli rilevanti nell'ambito di un più complesso sistema sociale, basato sul potere d'influenza della tecnica stessa. L'utilizzo della tecnica implica di per sé una totale riscrittura dell'intera sfera relazionale. Gli strumenti tecnici, dopo essere stati creati, possiedono un potenziale d'azione che corrisponde quasi totalmente all'idea di base che li ha visti nascere. Una sedia è stata pensata per sedersi. Può essere usata anche per offendere ma quest'uso è un totale stravolgimento del suo essere strumentale. Una bomba atomica invece è pensata per distruggere. Non ha nessun altro fine. L'utilizzo della tecnica implica una radicale trasformazione dei rapporti umani, questa trasformazione tende ad assimilare le persone alle cose. Da un punto di vista del singolo, le relazioni tendono ad assumere un carattere commerciale, movimento dato dal continuo moto dei sistemi economici in cui tutto tende a una riduzione a merce. Da un punto di vista sociale, la reificazione conduce inevitabilmente alla perdita dell' $\mathrm{i}$ dentità e alla massificazione. La massa non è un fatto numerico ma dipende dalla forma dei rapporti sociali.

${ }^{28}$ Cfr. E. Zolla, Eclissi dell'Intellettuale, Bompiani, Milano 1971, p.9. 
Si ha massa laddove l'indifferenza profonda regna fra persona e persona e i movimenti generali e particolari sono coordinati da forze esterne, per lo più anonime». ${ }^{29}(.$.$) «Il carattere della massa ondeggia fra l'isteria e la cupez-$ za e i sentimenti non hanno forma. ${ }^{30}$

Un tratto caratteristico della massa è il suo divorzio con il mondo della natura, un mondo dove il continuo mutare degli eventi naturali tiene lontana la noia di vivere. ${ }^{31}$

${ }^{29}$ Cfr. E. Zolla, op. cit., p.23.

${ }^{30} \mathrm{Ivi}$. p. 24

${ }^{31}$ Per quanto riguarda i principali riferimenti dell'opera di Zolla, essenziale è il lavoro di Marx ed Engels sull'alienazione, sul carattere di feticcio della merce, sulla condizione operaia. Lavori dei quali ci pare che Zolla utilizzi principalmente le intuizioni di carattere antropologico e di critica sociale (in particolare di Marx: Das Kapital. Kritik der politishen Ökonomie, 1867 (I Vol.), 1885-94 (II e III Vol.) e Ökonomish-philosophishe Manuskripte, 1932, e di Engels: Der Ursprusung der Familie des Privateigentums un des Staats, 1884, e Die Lage der arbeitenden Klasse in England nach eigner Anschauung und authentischen Quellen, 1845. Da sfondo costante è il lavoro di Freud, che Zolla utilizza da una profonda assimilazione del metodo analitico. Il termine Uomo-Massa è delineato per primo nell'opera di J. Ortega y Gassett, La rebelión de las masas, 1929. Importanti sono i riferimenti all'opera di W. Benjamin, Das Kunstwerk im Zeitalter seiner technischen Reproduzierbarkeit, 1936; lavoro in cui si concepiscono le tecniche moderne di riproduzione come una distruzione del concetto tradizionale di opera e in cui si denuncia la totale dipendenza dell'arte dalla politica. L'opera di G. Anderes, Die Antiquertheit des Menschen, 1957 è presa direttamente in considerazione. per aver posto l'accento sul carattere di continuo superamento dei limiti umani da parte delle macchine che hanno reso le performance degli uomini ridicole e obsolete e hanno generato nell'uomo, un senso di vergogna prometeica . Al lavoro di M. McLuhan, Myth and Mass Media, Zolla riconosce il merito di aver individuato la caratteristica dei mezzi di comunicazione di massa che non sono più dei soli mezzi ma, per la loro struttura stessa, sono già dei messaggi, dei modi di configurare la realtà. Importanti riferimenti sono le opere di Adorno, Dialektik der Aufklärung. Philosophische Fragmente, 1947, in cui si denuncia la deriva dell'illuminismo in pensiero mitico e il tentativo di dominare la natura si trasforma in dominio dell'uomo sull'uomo e in estraneità della natura all'uomo. Minima moralia. Reflexionen aus dem beschädigten Leben, 1951, in cui Adorno analizzando aspetti diversi della vita quotidiana individua i segni della nascita di un nuovo tipo umano, la freddezza borghese e l'atrofia di tutto ciò che è umano. Philosophie der neuen Musik, 1949 , in cui si sottolinea come in un mondo dominato dalla divisione del lavoro, la musica abbia perduto la sua autonomia. Importanti riferimenti sono all'opera di M. Weber, Die protestantische Ethik un der Geist des Kapitalismus, 1905 opera che Zolla utilizza come retroterra per svelare i meccanismi di «elezione» nella mentalità dell'Uomo-Massa . 


\section{L’impossibile ritorno}

Zolla non ha mai nascosto la sua posizione molto critica nei confronti della società della tecnica e dei consumi, che riduce l'uomo a uno strumento passivo di accorte manipolazioni. Nella sua opera, sino agli anni 70' soprattutto, ha cercato di porre l'accento sul rischio di riduzione dell'umano a pura cosa tra le cose. Zolla ha un'idea chiara della relazione tra uomo e natura:

L'uomo che ha voluto sottomettere la natura diventa schiavo di un apparato cieco, un atomo sociale votato all'inganno o alla disperazione: la scienza non è liberatoria perché vive dell'accecamento verso tutto ciò che esclude. ${ }^{32}$

Zolla sembra essere interessato (e questo lo accomuna ad Ortega) alla liberazione (possiamo dire alla salvezza della mia circostanza senza cadere nell'errore?) e non al dominio della materia e alla sottomissione del mondo naturale. Zolla conosce troppo bene le filosofie dell'estremo Oriente per non apprezzare e indirizzarsi alla via del Bodhisattva. Tuttavia, lungi dal disinteressarsi del mondo che lo circondava e pur interessato comunque a vivere le esperienze legate al numinoso, Zolla ha continuato per tutta la sua vita ad analizzare la società e il suo divenire, ponendosi sempre come un punto di riferimento per chi desiderava accostarsi allo studio e alla ricerca di percorsi «alternativi», rispetto alla retorica dei consumi e del possesso. In un articolo apparso su La Nazione, il 13 marzo del 1962, Zolla descrive un processo di distacco dalla società dei consumi, una forma di ascesi, che consiste in un progressivo allontanamento dagli oggetti fabbricati e in un ritorno alla natura:

Così un giovane educato, che sia padrone in casa propria, non avrà difficoltà a gioire di fare a meno della televisione e della radio, gustandosi l'intimità di un luogo silenzioso. Ma il piacere di sbarazzarsi di quella prima schiavitù ne attirerà una seconda: dopo un po' smetterà di entrare in un cinema. E abolirà via via tutte le umilianti soggezioni che negli ultimi cinquant'anni hanno irretito l'uomo comune. Dapprima proverà un certo malessere, come quello che assale i primi giorni all'arrivo in campagna appena ci si è liberati

\footnotetext{
${ }^{32}$ Cfr. E. Zolla, Gli orfani della metafisica, in Corriere della Sera, 7 giugno 1966, ora in E. Zolla, Gli arcani del potere. Elzeviri 1960-2000 (a cura di) G. Marchiano, BUR, Milano 2009, p.24.
} 
dagli assilli urbani: un intero sistema di difese nervose viene smobilitato a pezzo a pezzo prima che ci si possa immergere beatamente nella quiete. Poi, si respirerà meglio. Ma ce la farà il poverino ad arrestarsi a quei vantaggi senza sentire il richiamo di emigrare davvero in campagne oramai disertate, in villaggi montani abitati solo da anziani? A questo punto una Medusa, paurosa quanto quella da cui ha preso accurate distanze lo minaccerà da vicino: il dilemma della comunità agraria, con i beni in comune, col ritorno al patriarcato (o matriarcato), mai del tutto isolata dal mondo che la circonda e mai perfettamente edotta su chi è il caso di accogliere o escludere, su quali norme osservare. (...) Nascono problemi lunari del genere: fino a che punto è lecito ricorrere alle macchine? Vanno usati o no gli antibiotici, forse deleteri per l'integrità della stirpe ma così risolutivi per la guarigione dell'individuo. (...) Vagheggiare, ricreare in noi il passato preindustriale andrebbe accolto come una soluzione archi medica, un punto su cui far leva per rovesciare il peso delle costrizioni che ci schiacciano, non come un porto dove trovare un riposo mortuario. Una volta contrapposto il passato al presente, ci sarà un equilibrio perfetto, un vuoto privo di coercizione, dove ognuno troverà ciò che egli è destinato per vocazione intrinseca, senza mescolanza d'arbitrio o pressioni. (...) A questo punto si potrà anche sentirsi del tutto smarriti, ma proprio il fatto di vedere il vuoto come fonte di smarrimento e non invece uno spazio di libertà, sarà la prova se siamo davvero riusciti dalla coazione, se si è acquistata la spontaneità che il mondo ottunde. ${ }^{33}$

Zolla sa perfettamente che la natura antropologica dell'uomo di oggi è totalmente mutata rispetto a quella dell'uomo naturale, che era capace, di assimilare molto da poco e di vivere un contatto così immediato e diretto con la natura che oggi, anche volendo, ci sarebbe precluso. Un ritorno alla condizione naturale dell'uomo sarebbe, dunque, impossibile. Tuttavia, anche il semplice «vagheggiare» un ritorno al passato rappresenta un esercizio mentale in grado di bilanciare la tendenza incontrastata verso un'ideologia del progresso illimitato che, in fin dei conti, non convince neanche i suoi maggiori esponenti. ${ }^{34}$ Il vuoto e lo spaesa-

${ }^{33}$ Cfr. E. Zolla, Limpossibile ritorno, in La Nazione, 13 marzo 1963, ora in E. Zolla, Gli arcani del potere. Elzeviri 1960-2000 (a cura di) G. Marchiano, BUR, Milano 2009, p.91.

34 «Il M.I.T. aveva dato per scontato che nessuno avrebbe mai posto in dubbio l'idea del progresso: dalla scimmia era tutto un salire (con qualche sosta) sino al M.I.T. corona dell'evoluzione, tappa verso alte vette. Ma chi potrebbe dar credito ormai ad una fiaba simile salvo accecarsi e rifiutare la 
mento provocati dalla lotta fra queste due tendenze contrapposte (ritorno al passato e volontà di progresso), generano la condizione preliminare per pensare a una vera alternativa alla società della tecnica che rimane nell'opera di Zolla quella del Mistico. Il Mistico riproduce nella sua esperienza iniziatica le condizioni di vita di un mondo in cui la tecnica perde di potenza e di fascinazione, l'esperienza mistica consiste appunto nel riproporre lo stile di vita in cui si possono ripetere le:

[...]Condizioni di vita di una comunità diversa e più arcaica, fondata sulla coltivazione dell'orto o sull'attività che sostituisce in un mondo urbano la spigolatura delle tribù felici: la mendicità, la dove sia ripristinata la purezza dei costumi (voto di castità), la fusione delle volontà (voto di obbedienza) l'indifferenza al guadagno e all'accumulazioni di beni (voto di povertà). $[\ldots]$ il mistico torna a uno stato anteriore all'emanazione di leggi, quando il costume sorreggeva l'uomo senza che egli se ne avvedesse. ${ }^{35}$

Questa posizione, difesa e avvalorata da un lavoro di tutta una vita, può causare scandalo e perplessità. Qui, per utilizzare il linguaggio orteguiano, la via del Bodhisattva è seguita totalmente e di conseguenza (come aveva fatto notare Ortega) la tecnica che si sviluppa da questo progetto di vita è elementare e tutta in funzione della tecnica spirituale. Tutta la tecnica del Mistico è tecnica dell'anima rivolta alla salvezza:

L' "essere" di cui essi parlano deve essere una salvezza. Che esso sia uno, senza dualità, non diviso, senza attributi, senza come e senza modo (come entrambi dicono) non è un fatto meramente metafisico, ma anche un fatto di salvezza. E che l'anima sia eternamente una con l'eternamente uno, non è un fatto interessante scientificamente, ma qualcosa da cui dipende la salvezza dell'anima. Tutte le assicurazioni e le prove per la totale in distinzione, la completa semplicità. Lidentità assoluta, le prove e le declamazioni contro la molteplicità, la separazione, la dispersione — per quanto imitino l'ontologia razionale - sono per entrambi in ultima analisi significative, pro-

\footnotetext{
lunga serie di prove contrarie?». Cfr. Introduzione a William Irwin Thompson, All'orlo della storia, Rusconi, Milano 1972, ora in E. Zolla, Gli arcani del potere. Elzeviri 1960-2000 (a cura di) G. Marchiano, BUR, Milano 2009, p.112.

${ }^{35}$ Cfr. E. Zolla, I mistici dell'Occidente, Adelphi, Milano 2003, pp.22-24.
} 
prio perché trattano della salvezza. "La dove, anche per un attimo, v'è distinzione, la v'è pericolo, la grande miseria. ${ }^{36}$

Lo stesso Ortega non ha nascosto una certa ammirazione per i mistici e gli asceti, ritenuti:

I rarissimi individui che abbiamo riconosciuto capaci di uno sforzo spontaneo e aristocratico. Sono gli uomini eletti, i nobili, gli unici attivi, e non solo reattivi, per i quali vivere è perpetua tensione, incessante disciplina. $^{37}$

Tuttavia, sposare interamente questa posizione è impossibile per Ortega, e la sua principale preoccupazione rimane quella dell'uomo europeo: come collocarsi nell'ambito della società e vivere la vita nel migliore dei modi possibili. Per lo più, possiamo parlare di una preoccupazione politica, intendendo con questo termine la relazione tra individuo e polis, tra individuo e collettività.

\section{Ricapitolazione e conclusione}

Dopo aver comparato questi autori, diversi per cultura, età e riferimenti, ma accomunati da una volontà di comprendere i fenomeni della tecnica per coglierne la natura sostanziale di situazione, che muta nel tempo e che si adatta alle ambizioni dell'essere umano, possiamo procedere a una ricapitolazione. Ortega parte da una considerazione fondamentale: la tecnica si sviluppa in base al progetto che ogni individuo si pone di realizzare. L'ideale di uomo che ognuno vuole essere determina e produce tecniche diverse. Se si sposa un ideale di uomo che percepisce la realtà come una lotta e la natura come un luogo ostile da controllare e sottomettere, allora questo tipo di uomo svilupperà una tecnica aggressiva e potente, infinitamente complessa e che necessita molti anni di studio e si creerà un apparato produttivo in grado di generare tutti gli strumenti che sono necessari per il suo sviluppo. Al contrario, se l'ideale che l'uomo si propone di realizzare è quello di un sostanziale rispetto della natura e dei suoi ritmi e una vita umana degna e responsabile, in cui l'uomo non sottomette l'altro uomo, la tecnica che si svilupperà sarà

${ }^{36}$ Cfr. R. Otto, Mistica orientale, mistica occidentale, Marietti, Casale Monferrato 1985, p.25.

${ }^{37}$ Cfr. J. Ortega Y Gasset, La ribellione delle masse, SE, Milano 2001, p. 96. 
semplice ed essenziale. Non ci sarà bisogno di specialisti e l'apparato produttivo sarà facilmente gestibile con pochi sforzi. Ortega è consapevole che l'evoluzione storica ha abbracciato quasi totalmente il modello dello sviluppo tecnico illimitato, perché le ambizioni umane sembrano indirizzarsi sul modello del gentleman, piuttosto che sul Bodhisattva. Allora la possibilità di un'uscita da un mondo così minacciato dalla tecnica sembra essere quella offerta dall' hidalgo che non lavorando non crea tecnica. Però, non è meno determinato a dare alla propria condizione una soluzione degna. Ispirato dall'opera di Cervantes, che ha saputo offrire al mondo un ideale di opera in grado di salvare l'ideale mediterraneo dell'uomo e, allo stesso tempo, approfondire le ragioni esistenziali più importanti, Ortega si colloca nell'ambito della tradizione filosofica e considera la filosofia una possibilità di salvare la propria situazione e di conseguenza il proprio Io. E. Zolla, invece, critica in maniera radicale la tecnica, considerata una degenerazione rispetto alle qualità dell'uomo naturale e si spinge sino a proporre, come condizione normale dell'uomo, il mistico. La salvezza della propria situazione è nell'ascesi e nel ritorno alla condizione originaria della vita pre-industriale che ha ridotto l'uomo moderno a un mero soggetto di continua manipolazione in parte occulta. Entrambi gli autori hanno saputo porre l'accento sul legame esistente tra sviluppo tecnico e pensiero di massa ma poi, ognuno ispirato dalle proprie convinzioni più radicate, ha proposto soluzioni differenti. Più politica e filosofica quella di Ortega, più mistica e «orientale» quella di Zolla.

\section{Bibliografía}

\section{Opere di José Ortega y Gasset}

\subsection{Edizioni delle Opere Complete}

Obras completas (2004). 10 vols., Madrid: Taurus (en proceso de edición).

Obras completas (1983). 12 vols., Madrid: Alianza Editorial.

\subsection{Edizioni delle sue opere utilizzate}

La rebelión de las masas (2003). Edición de Domingo Hernández Sánchez, Madrid: Tecnos. La rebelión de las masas (1999). Edición de Thomas Mermall, Madrid: Castalia. Meditaciones del Quijote (1995). Edición de Julián Marías, Madrid: Cátedra. Meditación de la técnica y otros ensayos (1977). Madrid: Revista de Occidente. 


\section{Monografie e articoli utilizzati}

\subsection{Monografie}

Álvarez Gómez, M. E. (2003). Unamuno y Ortega. La búsqueda azarosa de la verdad, Madrid: Biblioteca Nueva.

CAMBI, F. (2007). Ortega y Gasset e la Bildung: studi critici, Milano: UNICOPLI.

Cangiotti, G. (1979). Ortega Y Gasset e la filosofia della tecnica, Bologna: Clueb.

Chaco Viu, V. (2000). Los intelectuales y la politica. Perfil público de Ortega y Gasset, Madrid: Biblioteca Nueva.

FERraCUTI, G. (1990). Jose Ortega y Gasset: esperienza religiosa e crisi della modernità, Rimini: Il Cerchio.

Ferrero Lavedán, I. (2005). La teoría social de Ortega y Gasset: los usos, Madrid, Biblioteca Nueva $\left(2^{\circ} \mathrm{Ed}\right)$.

Fosio M.,- Cipolloni M.,-Leveque. J.C. (cur.) (2001). Ortega y Gasset pensatore e narratore dell'Europa: Milano, 13-14 novembre 1988; Gargnano del Garda, 16-18 novembre 1998) Milano: Cisalpino.

Lasaga Medina, J., (2003). José Ortega y Gasset (1883-1955). Vida y filosofia, Madrid: Biblioteca Nueva.

LEVEQUE, J. C. (2003). Forme della ragione storica: la filosofia di Ortega y Gasset come filosofia europea, Pisa: Plus-Universita di Pisa.

Meregalli, F. (1995). Introduzione a Ortega y Gasset, Roma-Bari: Laterza.

Molinuevo, J.L. (2002). Para leer a Ortega, Madrid: Alianza Editorial.

SAN MARTIN, J. y LaSAGa, J (ed.). (2005) Ortega en circustancia: una filosofia para el siglo XXI, Madrid: Biblioteca Nueva/Fondacion Ortega y Gasset.

SAVIGNANO, A., (1996). Introduzione a Ortega y Gasset, Roma-Bari: Laterza.

SEVILlA, J. M. (2002) Ragione narrativa e ragione storica : una prospettiva vichiana su Ortega y Gasset, Perugia: Guerra.

VENTURINI, R. (2008) Ortega y Gasset: la realtà radicale e il sociale: osservazioni schutziane, Torino: Trauben.

Zamora Bonilla, J., (2001) Ortega y Gasset, Madrid: Plaza y Janès.

\subsection{Articoli}

Cabrera GarCia, M.I. (1994). Humanización versus técnica, pureza y elitismo. Un valor fundamental en la estética española de lo años 40, in Cuadernos de Arte, núm. XXV, Universidad de Granada, pp. 115-122. 
HARO HONRUBIA, A. (2004). Anális evolutivo de la idea de progreso: proyección actual de Meditación de la técnica de José Ortega y Gasset, en Revista de Estudios Orteguianos, núm. 8-9, Madrid, pp.185-219.

MORO ESTEBAN. P.L. (2001) La crisis del deseo. La rebeliión de las masas a la luz de Meditación de la técnica, en Revista de Estudios Orteguianos, n.2, Madrid, pp.215222.

\section{Opere di E. Zolla utilizzate}

L'eclissi dell'intellettuale (1959). Milano: Bompiani.

Volgarità e dolore (1962). Milano: Bompiani.

I mistici dell'Occidente (1963). Milano: Garzanti.

Le origini del trascendentalismo (1963). Roma: Edizioni di Storia e Letteratura.

Storia del fantasticare (1964). Milano: Bompiani.

Le potenze dell'anima (1968). Milano: Bompiani.

Che cos'è la tradizione (1971). Milano: Bompiani.

Un destino itinerante. Conversazioni tra Oriente e Occidente (1995), con Fasoli D., Venezia: Marsilio.

Archetipi (2005). Venezia: Marsilio.

Conoscenza religiosa. Scritti 1969-1983 (2006) (cur.). Marchianò G., Roma: Edizioni di Storia e

Letteratura.

Gli arcani del potere. Elzeviri 1960-2000 (2009). Intr. e cur. Marchianò G., Milano: Rizzoli Bur.

\subsection{Voci enciclopediche}

Simbologia, Enciclopedia Italiana Treccani, 1982 (vol.VI ).

Voce: Zolla Elémire di G.Marchianò, The Macmillan Encyclopedia of Religion, (2004) Cur. Lindsay Jones, $2^{\circ}$ ed., New York, vol. 14, pp. 9984-9987.

\subsection{Opere su E. Zolla}

\subsubsection{Convegni e incontri di studio}

Gli orienti del pensiero di Elémire Zolla:1926-2002. (2005/2006). Centro Pannunzio, Torino 16.XI.2002. Contributi critici di E. Albrile, F. Cuniberto, G. Filoramo, A. Grossato, P.Lucarelli, G.Marchianò, R.Mastromattei, S.Piano, S.Ronchey. Gli Atti sono in Elémire Zolla dalla morte alla vita, a c. di G. Marchianò, «Viator», Nuova Serie. 
Elémire Zolla e le virtù della scrittura, (2003) Club Popper, Fondazione Liberal, Montepulciano 21.VI.2003. Interventi di D. Fasoli, G. Marchianò, C. Médail, E. Rubin de Cervin Albrizzi. Gli Atti a c. di E. Gatta sono in parte raccolti in «Caffe Michelangelo", a.VIII, n.3, settembre-dicembre.

L'obbligo di sapere, la vanità di comprendere: Zolla e l'esoterismo. (2005). R.S.A.A., Università degli Studi La Sapienza, Roma 15.XI.2003. Intr. di C. Balacco Gabrieli, contributi critici di E. M. Baldacci, H. Cavallera, R. Mastromattei, P. Lucarelli, E. Gatta, A. Faj, M Abbaleo, G. Di Gesù, Bent Parodi di Belsito, G. Puglisi, G. Slonina Ubaldini. Gli Atti sono nel volume omonimo, $3^{\circ}$ Convegno Nazionale R.S.A.A. a c. di D. Cerniglia, Roma.

Filosofia perenne e intelletto d'amore nel pensiero di E. Zolla. (2006). Università di Bologna, a c. di Matteo Casari, 10.XI.2006. Presentazione di Carlo Gentili. Intervengono con G. Marchianò,

G. Azzaroni, F. Cuniberto e G. R. Franci.

Elémire Zolla. Un pensiero in cammino oltre il Novecento. (2006). Conversazione di G. Marchianò, Libreria Aseq, Roma 16.XI.2006.

La conoscenza degli archetipi. Omaggio a Elémire Zolla negli ottant'anni dalla nascita. (2006). Casa delle Letterature, Roma 29.XI.2006, a c. di M. I. Gaeta. Intervengono con G. Marchianò, P. Ricca, S. Ronchey e E. Trevi.

Elemire Zolla e l'idea di conoscenza religiosa nel suo lascito intellettuale. (2006). Gabinetto G. P. Vieusseux, Firenze,a c. di M. Bossi, 5.XII.2006. Coordina S. Givone. Interventi di Don A. Jacopozzi, G. Marchianò, M. Moneti.

Terre e cieli profondi. Elémire Zolla oltre il Novecento. (2006). Conversazione di G. Marchianò, ISUR, Scuola Superiore di Filosofia Indiana e Comparata, Rimini, 14.XII.2006, a c. di G. Ceccarelli e G. L. Magi.

Incontro col pensiero di E. Zolla nella sua città natale. (2007). Atrium, Torino,6.II.2007, a c. di L. Scarlini, Relazioni di G. Marchianò e S. Ronchey.

L'ordine sacro del cosmo: l'orizzonte smarrito. Posizioni a confronto Eliade-Zolla. (2007). relazione di G. Marchianò al Convegno: "Le forme della Tradizione, del simbolo e del sacro nei percorsi iniziatici della Libera Muratoria Universale», a c. di G. Seri, Antico e Primitivo Rito di Memphis e Misraim, Perugia, 15.XII.2007.

I doni dell'amico celato nel cuore. A proposito di un carteggio inedito di E. Zolla. (2008). Associazione Crocevia,Catania 15.III.2008, c. di R. Mondo e L. Turinese. Interventi di C. Guardo, R. Jannello, G. Marchianò e F. Battiato.

La tradizione spirituale in Italia: Zolla ritrovato. (2008). Il Vicariato, Castello di Pietrarubbia (PU) 7 giugno 2008, a c. di G. Marchianò. Interventi di Nuccio D’Anna, Mino Gabriele, Tamara Albertini, Don Alfredo Jacopozzi, A. Colimberti, A. Amisano, R. Moretti e le catalogatrici della biblioteca Zolla: Elena Cheli e Adriana Alessandrini. 
L'arte del risveglio e l'ascesi marziale. (2010). I Seminario Zolla A.I.R.E.Z., Lucignano d'Arezzo, 29-30 maggio 2010. Introduzione di G. Marchianò. Relazioni di: Don Alfredo Jacopozzi, "L'arte del risveglio nella mistica cristiana»; Grazia Marchianò, "Il risveglio alla consapevolezza nell'opera-specchio di Elémire Zolla»; M.o Massimo Cavazza e allievi, «Le tre virtù del pugno», sessione dimostrativa dello stile marziale wing chun.

\subsubsection{Tesi di Laurea}

La posizione sull'estetica filosofica e sull'estetica filmica di Elémire Zolla. (1972/73). Di G. Sammartino, relatore Prof. G. A. Bianca, Università di Catania, A.A. 1972-73.

Eclissi del sacro e pensiero mistico in Elémire Zolla. (1988/89). Di A. Marmo, relatore prof. F. Lazzari, Università di Salerno, A.A. 1988-1989.

Critica della civiltà e sincretismo nell'opera di Elémire Zolla. (1990-1991). Di A. Marra, relatore prof. A. Agnoletto, Università di Milano, A.A. 1990-1991.

Critica della cultura e sincretismo mistico nel pensiero religioso di Elémire Zolla. (1994-95) Di R. Valentini, relatore Prof.ssa F. Brezzi, III Università di Roma, a. a. 1994 - 95.

Elémire Zolla come fondatore di «Conoscenza religiosa». (1998/99). Di M. Cella, relatore prof. G. Casadio, Università di Salerno, A.A.1998-99.

Tradizione e "esperienza metafisica» nel pensiero di Elémire Zolla. (2002/03). Di F. Fusella, relatore Prof. M. Malaguti, Università di Bologna, A.A. 2002-2003.

Dall'anima allo spirito. Cristina Campo e Elémire Zolla: mistica e spiritualità nella svolta religiosa dei primi anni Sessanta. (2004/05). Di M. Lepore, relatore Prof. F. Michelini Tocci, Università Ca’Foscari di Venezia, A.A. 2004-2005.

Eclissi dell'intellettuale. Aspetti dell'estetica di Elémire Zolla, (2007-2008). Di Sandro Rizzitelli, Relatore Prof. M.Perniola, Università di Roma Tor Vergata, A.A. 2007-2008.

Elémire Zolla postumo. (2008/9). di A. Amisano, relatore Prof. N. Spineto, Università di Torino, A.A. 2008-2009 (in corso d'opera).

\subsubsection{Monografie e articoli}

GATTA, E. (2003). Elémire Zolla (1926-2002): I giorni le opere. "Caffe Michelangelo», a.VIII, n.3, settembre-dicembre.

MARCHIANÒ, G. (2005/06). Elémire Zolla dalla morte alla vita. Fascicolo monografico negli ottanta anni dalla nascita "Viator», Nuova Serie.

- (2002). Elémire Zolla: Sprazzi di una biografia interiore. "Viator», a.VI.

- (2006). Il conoscitore di segreti. Una biografia intellettuale. Milano: Rizzoli.

- (1991). La religione della terra. Como: Red.

Recibido: 4/04/2011

Aceptado: 13/07/2011 